\title{
Martensitic transition assisted modification of the antiferromagnetic ordering in Ge-site doped MnNiGe alloys
}

S. C. Das, J. Sannigrahi, P. Dutta, S. Pramanick, D. Khalyavin, D. T. Adroja, and S. Chatterjee

\section{Published version information}

Citation: SC Das et al. "Martensitic transition assisted modification of the antiferromagnetic ordering in Ge-site doped MnNiGe alloys." Phys Rev B 103, no. 9 (2021): 094422.

DOI: $\underline{10.1103 / P h y s R e v B .103 .094422}$

This version is made available in accordance with publisher policies. Please cite only the published version using the reference above. This is the citation assigned by the publisher at the time of issuing the APV. Please check the publisher's website for any updates. 


\title{
Martensitic transition assisted modification of the antiferromagnetic ordering in Ge-site doped MnNiGe alloys
}

\author{
S. C. Das, ${ }^{1}$ J. Sannigrahi, ${ }^{2,3}$ P. Dutta $\odot,{ }^{1,4}$ S. Pramanick, ${ }^{1}$ D. Khalyavin, ${ }^{2}$ D. T. Adroja,,${ }^{2,5}$ and S. Chatterjee $\oplus^{1, *}$ \\ ${ }^{1}$ UGC-DAE Consortium for Scientific Research, Kolkata Centre, Sector III, LB-8, Salt Lake, Kolkata 700 106, India \\ ${ }^{2}$ ISIS Facility, Rutherford Appleton Laboratory, Chilton, Didcot OX11 OQX, United Kingdom \\ ${ }^{3}$ Physics of Quantum Materials, Max Planck Institute for Chemical Physics of Solids, \\ Nothnitzer Strasse 40, 01187 Dresden, Germany \\ ${ }^{4}$ New Chemistry Unit and School of Advanced Materials, \\ Jawaharlal Nehru Centre for Advanced Scientific Research, Jakkur, Bangalore 560064, India \\ ${ }^{5}$ Highly Correlated Matter Research Group, Physics Department, \\ University of Johannesburg, P.O. Box 524, Auckland Park 2006, South Africa
}

(Received 29 January 2021; accepted 1 March 2021; published 15 March 2021)

\begin{abstract}
The magnetic equiatomic alloys of the nominal compositions $\mathrm{MnNiGe}_{0.9} \mathrm{Al}_{0.1}$ and $\mathrm{MnNiGe}_{0.928} \mathrm{Ga}_{0.072}$ have been investigated through the neutron powder diffraction (NPD) technique. Both these alloys undergo a martensitic phase transition from the high-temperature hexagonal $\left(\mathrm{Ni}_{2} \mathrm{In}\right.$ type, with space group $\left.P 6_{3} / m m c\right)$ austenite phase to the low-temperature orthorhombic (TiNiSi type, with space group Pnma) martensite phase. Our NPD analysis confirmed an incommensurate antiferromagnetic ordering of the low-temperature martensitic phase with helical modulation. The incommensurate propagation vector $\mathbf{k}$ is found to be $(0.2065(1), 0,0)$ and $(0.2088(4), 0,0)$ for $\mathrm{Al}$ and $\mathrm{Ga}$ doped alloys, respectively, at $1.5 \mathrm{~K}$ and shows a monotonic increase with increasing sample temperature. On the other hand, a commensurate antiferromagnetic ordering was observed for the high-temperature austenite phase. The incomplete martensitic transition allowed both these incommensurate and commensurate antiferromagnetic structures to coexist down to the lowest temperature of measurement (1.5 K).
\end{abstract}

DOI: 10.1103/PhysRevB.103.094422

\section{INTRODUCTION}

Materials with concurrent magnetic and structural (martensitic) transitions are widely known for showing diverse properties compared to materials with naturally separated magnetic and structural transition temperatures [1-7]. Consequently, a regime of first-order magnetic transition with the characteristic of overlapping magnetic and martensitic phase transition (MPT) gets priority for macroscopic as well as microscopic research. Among the various members of the magnetic equiatomic family (general formula $M M^{\prime} X$, where $M$ and $M^{\prime}$ are transition metals and $X$ is a nonmagnetic $s p$ element), MnNiGe is widely accepted as a promising candidate for practical applications as it possess multiple functional properties, including the shape memory effect, a large magnetocaloric effect, magnetoresistance, the exchange bias effect, etc. [8-23]. In addition, the undoped MnNiGe alloy undergoes multiple phase transformations (structural and magnetic depending on the thermal state of the material), namely, (i) MPT around $470 \mathrm{~K}$ from the high-temperature $\mathrm{Ni}_{2} \mathrm{In}$-type hexagonal austenite phase (space group $P 6_{3} / \mathrm{mmc}$ ) to the TiNiSi-type orthorhombic martensite phase (space group Pnma) and (ii) a paramagnetic (PM) to spiral antiferromagnetic (AFM) transition below or around $346 \mathrm{~K}$ [8-10,16,24-28].

*souvik@alpha.iuc.res.in
As is evident from previous studies, transition temperatures in $\mathrm{MnNiGe}$ are highly sensitive to the compositional elements and their molar percentage [8-23]. Partial substitution of different sites of the parent compound with a suitable foreign element corresponds to a significant change in both structural and magnetic transition temperatures [8-23]. Interestingly, the alloy retains the AFM ordering after doping at the $\mathrm{Mn} / \mathrm{Ni}$ site, as confirmed by the magnetic and neutron powder diffraction (NPD) analysis [9,10,15-19,24-28]. On the other hand, the effect of doping at the Ge site is slightly different than $\mathrm{Mn} / \mathrm{Ni}$ site doping. Ge-site doped alloys undergo a PM austenite to ferromagnetic (FM) austenite transition, followed by an AFM martensite transition during cooling [11-14]. However, the claim of the FM austenite phase is entirely based on the macroscopic measurements, and corresponding confirmative microscopic studies have yet to be explored. Therefore, it is pertinent to investigate the detailed magnetic structure of Ge-site doped alloys and its thermal evolution through neutron diffraction studies to unveil the true nature of mutual correlation among structural, electronic, and magnetic degrees of freedom in these compounds.

For doping analysis, the size of the doping agent (dopant) has a potent role in the material formation and on its properties. In this work, we study two Ge-site doped alloys with different dopant sizes compared to Ge, namely, (i) $\mathrm{MnNiGe}_{0.9} \mathrm{Al}_{0.1}$ (smaller $\mathrm{Al}$ doping) and (ii) $\mathrm{MnNiGe}_{0.928} \mathrm{Ga}_{0.072}$ (larger Ga doping) through NPD analysis. Both these alloys are reported to undergo the transition 
sequence AFM martensite $\rightarrow$ FM austenite $\rightarrow$ PM austenite during heating from $1.5 \mathrm{~K}$ [11-14]. However, our neutron diffraction analysis confirms the absence of the appearance of any ferromagnetically ordered austenite phase in these alloys. In contrast, a helically ordered incommensurate AFM martensite (with an orthorhombic structure) transformed to commensurate AFM austenite (with a hexagonal structure) during heating for both the doped alloys. The AFM ordering strength in the martensitic phase has also been enhanced with temperature, which is evident from the monotonic increase in the incommensurate propagation vector. Being in the AFM austenite phase, a further increase in temperature leads to direct AFM to PM transition without any structural modification for both alloys.

\section{EXPERIMENTAL DETAILS}

\section{A. Synthesis and magnetic characterization}

The $\mathrm{Al}$ and $\mathrm{Ga}$ doped $\mathrm{MnNiGe}$ polycrystalline alloys of the nominal compositions $\mathrm{MnNiGe}_{0.9} \mathrm{Al}_{0.1}$ and $\mathrm{MnNiGe}_{0.928} \mathrm{Ga}_{0.072}$ were prepared by arc melting the constituent elements (with purity $>99.9 \%$ ) in an inert argon atmosphere. We repeated the melting process four times to achieve the desired homogeneity of the prepared alloys. The ingots were then sealed in an evacuated quartz capsule and annealed at $800^{\circ} \mathrm{C}$ for $100 \mathrm{~h}$, followed by rapid quenching in ice water. Henceforth, we shall refer to the alloy $\mathrm{MnNiGe}_{0.9} \mathrm{Al}_{0.1}$ as MNGA and the alloy $\mathrm{MnNiGe}_{0.928} \mathrm{Ga}_{0.072}$ as MNGG. The dc magnetization $M$ of the present samples was measured using a commercial cryogen-free high magnetic field system from Cryogenic Ltd. (magnetic field $H$ range of $\pm 150 \mathrm{kOe}$ and temperature $T$ range of $2-300 \mathrm{~K}$ ). The samples were tightly packed, and $33.4 \mathrm{mg}$ of MNGA and $43.1 \mathrm{mg}$ of MNGG were used during the magnetic measurements.

\section{B. Neutron powder diffraction}

The NPD measurements of the presently studied alloys were carried out in the cold neutron (time of flight) diffractometer WISH at the ISIS Facility (Rutherford Appleton Laboratory) in the United Kingdom [29]. A 6-mm-diameter vanadium cell was used to mount the powder samples. The entire vanadium cell was placed in a standard liquid He cryostat to decrease the sample temperature down to $1.5 \mathrm{~K}$. Data were collected at different constant temperatures $T$ in the warming cycle. We used about $5 \mathrm{~g}$ of each alloy for NPD measurements. For magnetic structure determination, we recorded five long scans (each scan was $30 \mathrm{~min}$ in duration) below, around, and above the MPT. In addition, several quick scans (10-min duration each) were performed for order parameter determination. Notably, the neutron scattering cross section of the constituent elements of the present alloys are $\mathrm{Mn} \rightarrow$ 2.15(3) barns, $\mathrm{Ni} \rightarrow$ 18.5(3) barns, Ge $\rightarrow$ 8.60(6) barns, $\mathrm{Ga} \rightarrow 6.83(3)$ barns, and $\mathrm{Al} \rightarrow 1.503(4)$ barns [30]. Rietveld refinement of the recorded NPD data was performed using the FULLPROF software package [31]. We used the BASIREPS, GSAS, VESTA software packages to create and visualize the model.
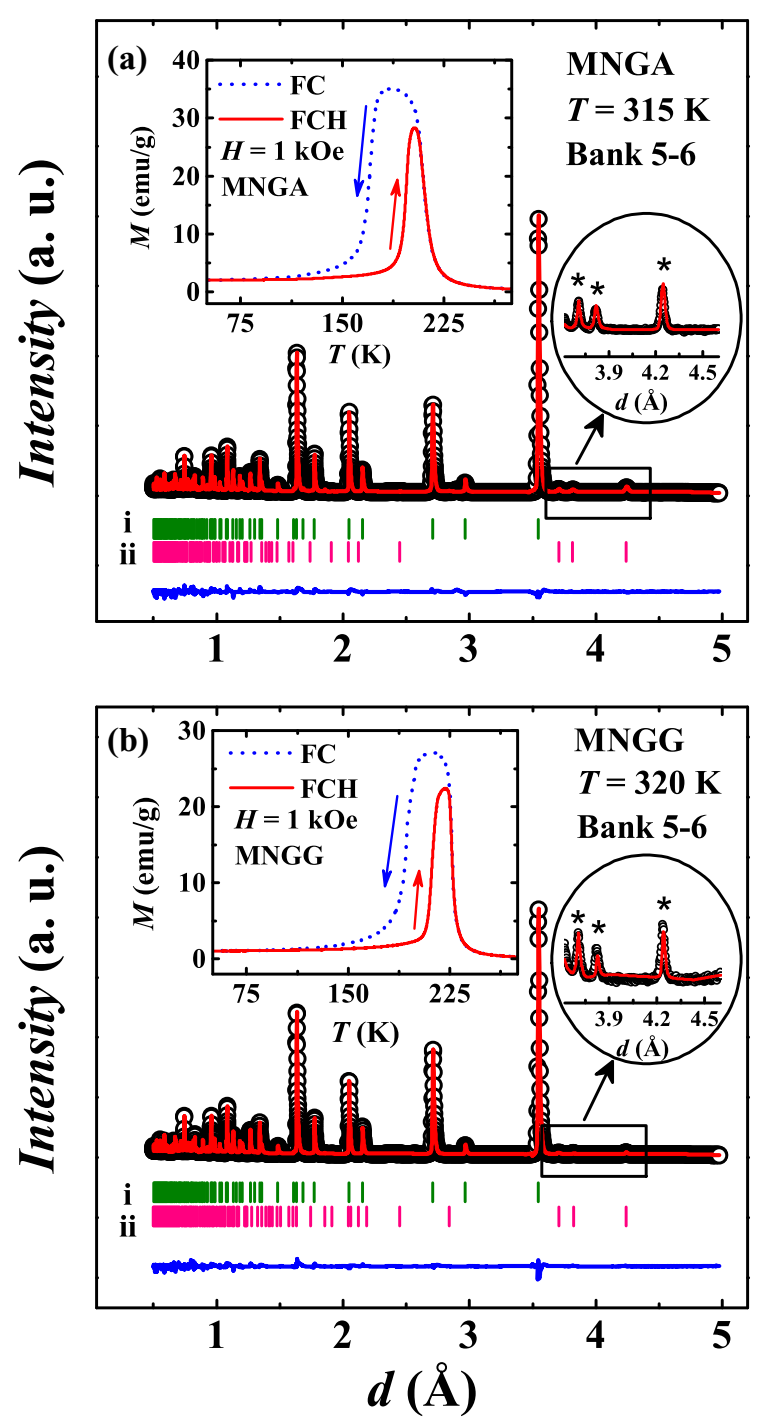

FIG. 1. (a) and (b) NPD data from detector banks 5 and 6 of WISH for $\mathrm{MnNiGe}_{0.9} \mathrm{Al}_{0.1}$ (MNGA) and $\mathrm{MnNiGe}_{0.928} \mathrm{Ga}_{0.072}$ (MNGG) alloys recorded at 315 and $320 \mathrm{~K}$, respectively. Here, black circles, red lines, and blue lines (lowermost line in the main panels) indicate the observed, calculated, and difference patterns, respectively. The first (olive, labeled i), and second (magenta, labeled ii) vertical bars indicate the peak positions for the $\mathrm{Ni}_{2}$ In-type (for MNGA and MNGG) and $\mathrm{MgZn}_{2}$-type (for $\mathrm{MnNi}_{1.3} \mathrm{Ge}_{0.7}$ ) hexagonal nuclear phases, respectively. Circular insets show an enlarged version of the respective NPD pattern for better visibility of the reflections originating from the $\mathrm{MnNi}_{1.3} \mathrm{Ge}_{0.7}$ composition (indicated by stars). The temperature $T$ variation of dc magnetization $M$ recorded in field-cooling $(\mathrm{FC})$ and field-cooled heating $(\mathrm{FCH})$ protocols in the presence of $1 \mathrm{kOe}$ of an external magnetic field $H$ are plotted in the square insets.

\section{EXPERIMENTAL RESULTS}

\section{A. Room temperature neutron diffraction}

The NPD data recorded around room temperature for the MNGA and MNGG alloys are presented in the main panels of Figs. 1(a) and 1(b), respectively. Rietveld refinement analyses of these NPD data indicate that both alloys crystallize in the $\mathrm{Ni}_{2} \mathrm{In}$-type hexagonal structure with space group 
$P 6_{3} / m m c$. In addition to the reflections associated with the $\mathrm{Ni}_{2}$ In-type hexagonal structure, a few additional reflections in the NPD patterns have also been noticed for both alloys. Such additional reflections are due to the presence of the $\mathrm{MgZn}_{2}$-type hexagonal $\mathrm{MnNi}_{1.3} \mathrm{Ge}_{0.7}$ alloy along with the presently studied MNGA and MNGG alloys. An enlarged view of the reflections coming from the $\mathrm{MnNi}_{1.3} \mathrm{Ge}_{0.7}$ phase, along with the calculated patterns, are shown in the circular insets in Figs. 1(a) and 1(b). The formation of the $\mathrm{MnNi}_{1.3} \mathrm{Ge}_{0.7}$ alloy during the preparation of magnetic equiatomic alloys is not uncommon and has already been reported by Kuźma et al. [32]. No structural or magnetic phase transitions are reported to be associated with the $\mathrm{MnNi}_{1.3} \mathrm{Ge}_{0.7}$ alloy. Our analysis indicates the presence of about 1.61 $( \pm 0.17)$ and $0.60( \pm 0.04)$ wt $\%$ of the $\mathrm{MnNi}_{1.3} \mathrm{Ge}_{0.7}$ phase in the MNGA and MNGG alloys, respectively. The lattice parameters obtained around room temperature for the MNGA $\left[a_{\mathrm{h}}=4.093(1) \AA, c_{\mathrm{h}}=5.415(8) \AA\right]$, MNGG $\left[a_{\mathrm{h}}=4.095(4) \AA, c_{\mathrm{h}}=5.420(7) \AA\right]$, and $\mathrm{MnNi}_{1.3} \mathrm{Ge}_{0.7}\left[a_{\mathrm{h}}=\right.$ $\left.4.890(7) \AA, c_{\mathrm{h}}=7.623(9) \AA\right]$ alloys match the previously reported data well [11-14,32]. No magnetic satellite reflection has been observed in any of the studied alloys around room temperature.

\section{B. dc magnetization}

To verify the presence of MPT in the presently studied MNGA and MNGG alloys, we recorded the $T$ variation of dc $M$ in the presence of $1 \mathrm{kOe}$ of an external $H$ in the fieldcooling (FC) and field-cooled heating (FCH) protocols [see square insets in Figs. 1(a) and 1(b)]. A restricted region of the $M(T)$ curves is plotted for clear visualization. On heating from $5 \mathrm{~K}$, the $M$ value remains almost unchanged up to $175 \mathrm{~K}(185 \mathrm{~K})$ for the MNGA (MNGG) alloy, followed by a sharp upturn in it, signifying the MPT. A further increase in sample temperature results in a sharp fall in the $M$ value around 205 and $224 \mathrm{~K}$ for MNGA and MNGG, respectively. This transition is associated with the magnetically ordered austenite state to the PM austenite phase. Thermal hysteresis associated with FC and FCH data around the MPT confirms the first-order nature of the transition. The observed range of MPT temperatures matched the previously reported data well [11-13].

\section{Identification of magnetic structure}

Now, let us concentrate on the present alloys' magnetic structures at the lowest temperature of the measurement. The 1.5 K NPD data confirm that both the MNGA and MNGG alloys show the orthorhombic crystal structure. However, along with the orthorhombic nuclear reflections, some additional nuclear (for the high- $T$ hexagonal phase and $\mathrm{MnNi}_{1.3} \mathrm{Ge}_{0.7}$ phase) and magnetic satellite reflections have also been noticed. The NPD data recorded at $1.5 \mathrm{~K}$ for the MNGA and MNGG alloys for different lattice plane spacing $d$ ranges are presented in Figs. 2(a)-2(d). Our analysis confirms that the weight percent of the $\mathrm{MnNi}_{1.3} \mathrm{Ge}_{0.7}$ phase observed for both the MNGA and MNGG alloys at $1.5 \mathrm{~K}$ is similar to that of the room temperature data. On the other hand, we noticed about $14.04( \pm 0.41)$ and $1.37( \pm 0.05)$ wt $\%$ of the high- $T$ hexagonal phase at the lowest $T$ of the measurement $(1.5 \mathrm{~K})$ for the MNGA and MNGG alloys, respectively. Apart from the nuclear reflections, several magnetic satellite reflections are also clearly visible, with the most intense magnetic reflection appearing at a very large value of $d(\sim 29.12 \AA$ for MNGA and $\sim 28.78 \AA$ for MNGG). The larger value of the magnetic form factor of Mn at larger $d$ or smaller $Q\left(=\frac{2 \pi}{d}\right)$ is the key reason for such an observation. Two different types of magnetic reflections have been identified for the MNGA alloy, namely, (i) the magnetic reflections that can be indexed by the incommensurate propagation vector $\mathbf{k}_{\mathbf{o}}=\left(k_{a}, 0,0\right)$, with $k_{a}=0.2065(1)$, having orthorhombic structure, and (ii) the magnetic reflections that can be indexed by the commensurate propagation vector $\mathbf{k}_{\mathbf{h}}=(0,0,0)$ having hexagonal structure. On the other hand, no traces of the magnetic satellite reflection have been observed for the commensurate AFM structure in the MNGG alloy. This may be due to the smaller percentage $[\sim 1.37 \%( \pm 0.05 \%)]$ of the high- $T$ hexagonal phase present at $1.5 \mathrm{~K}$ for the MNGG alloy. However, the magnetic reflections due to the incommensurate AFM structure can be indexed by using the propagation vector $\mathbf{k}_{\mathbf{o}}=\left(k_{a}, 0,0\right)$, with $k_{a}=$ $0.2088(4)$. Considering all nuclear and magnetic phases, we have successfully refined the NPD data for both the MNGA and MNGG alloys at $1.5 \mathrm{~K}$. Various refinement parameters at $1.5 \mathrm{~K}$ for both alloys are shown in Table I.

The magnetic structure which has been found to provide the best fitting quality for the incommensurate magnetic intensities involves spins localized on the Mn sites and rotating within the $b c$ plane (see Table II). The set of symmetry elements of the orthorhombic Pnma space group, which keep the incommensurate propagation vector $\mathbf{k}_{\mathbf{o}}$ unchanged (little group), splits the $4 c \mathrm{Mn}$ position into two orbits. The Mn sites from orbits 1 and 2 are denoted as $\mathrm{Mn}_{11}, \mathrm{Mn}_{12}$ and $\mathrm{Mn}_{21}, \mathrm{Mn}_{22}$, respectively, in Table II. As the $x$ coordinates of the Mn sites within the same orbit are found to differ by $\frac{1}{2}$, we have fixed the corresponding magnetic phases to $\frac{k_{a}}{2}$ during the refinement procedure. The relative magnetic phase between the orbits is not fixed by symmetry and was freely refined along with the size of the magnetic moments. The latter was constrained to be the same for both orbits. The parameters refined at $T=1.5 \mathrm{~K}$ for both the MNGA and MNGG alloys are summarized in Table II. As per the present model, the angle between the two adjacent Mn spins are found to be $\alpha \approx 37.17^{\circ}$ and $37.59^{\circ}$ for the MNGA and MNGG alloys, respectively, at $1.5 \mathrm{~K}$ [see Fig. 3(b)]. Some previous works on the $\mathrm{Mn} / \mathrm{Ni}$-site doped $\mathrm{MnNiGe}$ alloys also reported a similar helical magnetic structure of the low- $T$ martensite phase [25-28]. On the other hand, a simple collinear AFM structure has been observed for the magnetically ordered hexagonal austenite phase. Both magnetic structures are depicted in Figs. 3(a)-3(c).

\section{Temperature evolution of magnetic structure}

To probe the temperature evolution of the observed magnetic structure, we recorded NPD data at different constant $T$ during warming from $1.5 \mathrm{~K}$. Some restricted regions of the NPD patterns recorded at different constant $T$ for the presently studied alloys are presented by the contour plot in Fig. 4 (restricted areas are plotted here for better visibility 

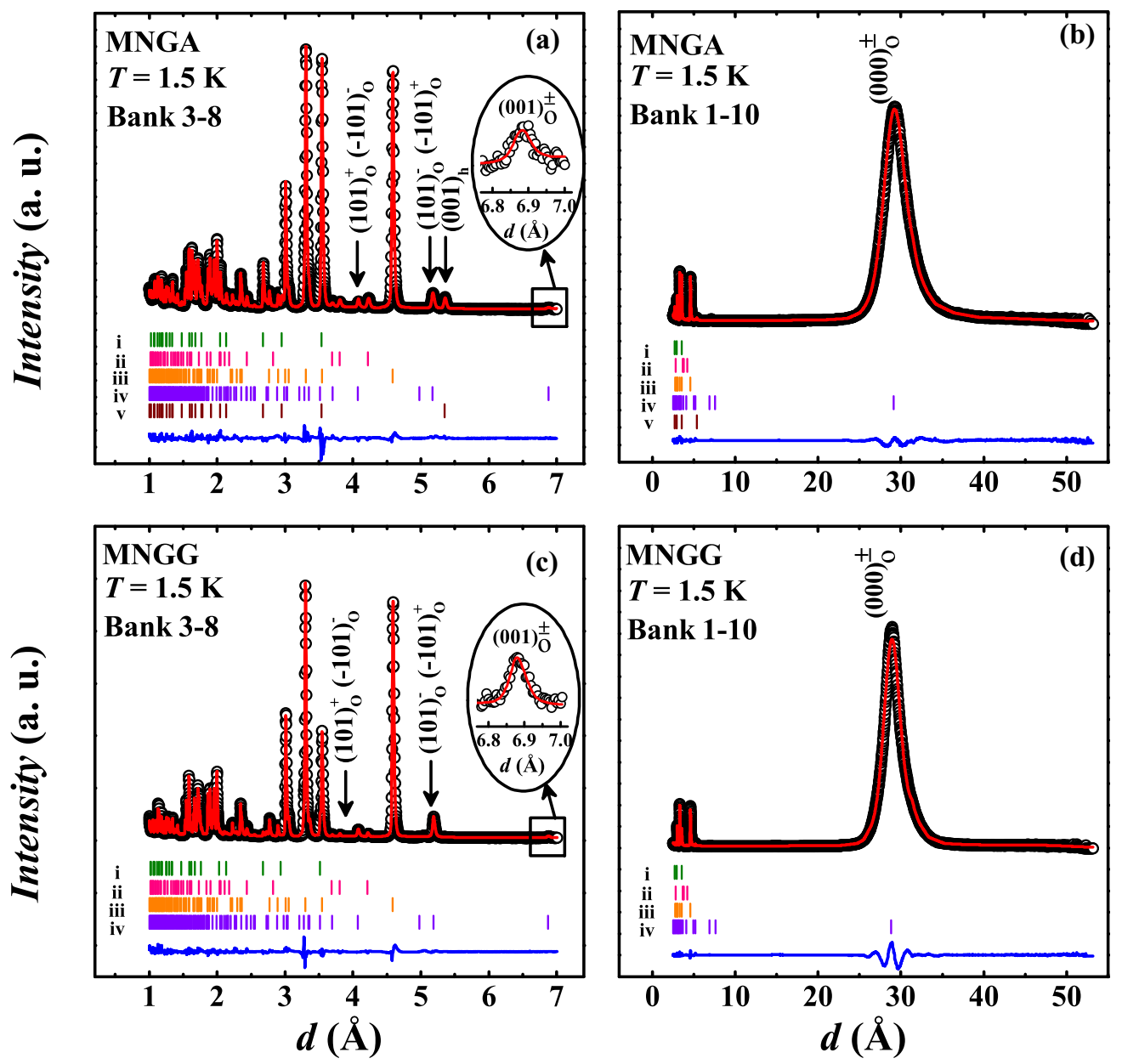

FIG. 2. NPD data recorded at $1.5 \mathrm{~K}$ in ambient conditions are plotted for the (a) and (b) MNGA and (c) and (d) MNGG alloys for two different ranges of lattice spacing $d$ from detector banks 3-8 and 1-10 of WISH. Insets in (a) and (c) show an enlarged view of the (001) o $^{ \pm}$ magnetic peak. Here, black circles, red lines, and blue lines indicate the observed, calculated, and difference patterns, respectively. The first (olive, labeled i), second (magenta, labeled ii), third (orange, labeled iii), fourth (purple, labeled iv), and fifth (wine, labeled v) vertical bars indicate the peak positions for the $\mathrm{Ni}_{2}$ In-type hexagonal nuclear phase (for MNGA/MNGG), $\mathrm{MgZn}_{2}$-type hexagonal nuclear phase (for $\mathrm{MnNi}_{1.3} \mathrm{Ge}_{0.7}$ ), orthorhombic nuclear phase, orthorhombic magnetic phase, and hexagonal magnetic phase (for MNGA), respectively.

of the magnetic satellite reflections). Apart from the structural transition, an increase in sample temperature results in some significant effects on the magnetic satellite reflections for both incommensurate and commensurate AFM structures, namely, (i) shifting of $(101)_{\mathrm{o}}^{+},(-101)_{\mathrm{o}}^{-}$, and $(000)_{\mathrm{o}}^{ \pm}$reflections towards the lower $d$, (ii) shifting of $(101)_{\mathrm{o}}^{-},(-101)_{\mathrm{o}}^{+}$, and $(001)_{\mathrm{h}}$ reflections towards the higher $d$, (iii) a sluggish decrease of the intensity of incommensurate AFM reflections with an initial increase in $T$ followed by a sharp decrease around the MPT, and (iv) unchanged intensity of the commensurate AFM reflection up to the MPT followed by a peaklike behavior with a further increase in $T$ that eventually vanishes above the magnetic transition $T$ (due to the increase in the $\mathrm{Ni}_{2}$ In-type hexagonal phase fraction around the MPT).

After successfully refining all the recorded NPD data using the same magnetic structures (an incommensurate helicoidal structure for the orthorhombic phase and a commensurate AFM structure for the hexagonal phase), we have noticed a monotonic increase in orthorhombic and hexagonal lattice parameters for both alloys (see Fig. 5). However, a slight slope change has been observed in the hexagonal lattice parame- ters around the magnetic ordering temperature of the high- $T$ hexagonal phase for both alloys [see Figs. 5(b) and 5(d)]. Successful refinement of NPD data at different constant $T$ also helped us to determine the phase fractions of the hexagonal ( $\mathrm{Ni}_{2}$ In-type), orthorhombic (TiNiSi-type), and $\mathrm{MnNi}_{1.3} \mathrm{Ge}_{0.7}$ $\left(\mathrm{MgZn}_{2}\right.$-type hexagonal) phases at different constant temperatures. Variations of such phase fractions as a function of $T$ for both alloys are plotted in Figs. 6(a) and 6(c), where a signature of MPT from the orthorhombic to hexagonal phase is clearly visible. The region of MPT observed from the NPD data matches the dc magnetization data well (between 150 and $240 \mathrm{~K}$ for MNGA and between 195 and $245 \mathrm{~K}$ for MNGG). Notably, the phase fraction of $\mathrm{MnNi}_{1.3} \mathrm{Ge}_{0.7}$ remains unchanged throughout the entire temperature range of the measurement. On the other hand, MPT is incomplete for both these alloys, which is clear from the presence of a high- $T$ hexagonal phase fraction along with the orthorhombic phase fraction below the MPT region $[\sim 14.04 \%( \pm 0.41 \%)$ for MNGA and $\sim 1.37 \%( \pm 0.05 \%)$ for MNGG].

We have also calculated and plotted the variation of the integrated intensity of the $(000)_{\mathrm{o}}^{ \pm}$and $(001)_{\mathrm{h}}$ magnetic 
TABLE I. Various structural parameters for the $\mathrm{MnNiGe}_{0.9} \mathrm{Al}_{0.1}$ (MNGA) and $\mathrm{MnNiGe}_{0.928} \mathrm{Ga}_{0.072}$ (MNGG) alloys at $1.5 \mathrm{~K}$ and around room temperature ( 315 and $320 \mathrm{~K}$ for the MNGA and MNGG alloys, respectively). Along with the weight percent of different phase fractions mentioned for different temperatures, there exists a $\mathrm{MnNi}_{1.3} \mathrm{Ge}_{0.7}$ phase with the following weight percentages: (i) for $\mathrm{MNGA}, 1.61 \%$ ( $\pm 0.17 \%$ ) at $1.5 \mathrm{~K}$ and $1.61 \%( \pm 0.11 \%)$ at $315 \mathrm{~K}$ and (ii) for MNGG, $0.58 \%( \pm 0.06 \%)$ at $1.5 \mathrm{~K}$ and $0.60 \%( \pm 0.04 \%)$ at $315 \mathrm{~K}$.

\begin{tabular}{|c|c|c|c|c|c|c|c|c|c|c|c|}
\hline Type & Site & $x$ & $y$ & $z$ & $B_{\text {iso }}$ & Type & Site & $x$ & $y$ & $z$ & $B_{\text {iso }}$ \\
\hline \multicolumn{12}{|c|}{ TiNiSi-type orthorhombic structure with space group $P n m a^{\mathrm{a}}$} \\
\hline $\mathrm{Mn}$ & $4 c$ & $0.032(6)$ & 0.250 & $0.180(2)$ & $1.30(5)$ & $\mathrm{Mn}$ & $4 c$ & $0.032(6)$ & 0.250 & $0.182(4)$ & $1.48(8)$ \\
\hline $\mathrm{Ni}$ & $4 c$ & $0.147(4)$ & 0.250 & $0.550(8)$ & $1.85(1)$ & $\mathrm{Ni}$ & $4 c$ & $0.146(6)$ & 0.250 & $0.557(5)$ & $1.21(4)$ \\
\hline \multicolumn{12}{|c|}{$\mathrm{Ni}_{2}$ In-type hexagonal structure with space group $P 6_{3} / m m c^{\mathrm{b}}$} \\
\hline $\mathrm{Mn}$ & $2 a$ & 0.000 & 0.000 & 0.000 & $2.37(8)$ & $\mathrm{Mn}$ & $2 a$ & 0.000 & 0.000 & 0.000 & $2.37(8)$ \\
\hline $\mathrm{Ni}$ & $2 d$ & 0.333 & 0.666 & 0.750 & $0.07(2)$ & $\mathrm{Ni}$ & $2 d$ & 0.333 & 0.666 & 0.750 & $0.07(2)$ \\
\hline $\mathrm{Ge}$ & $2 c$ & 0.333 & 0.666 & 0.250 & $0.61(9)$ & $\mathrm{Ge}$ & $2 c$ & 0.333 & 0.666 & 0.250 & $0.61(9)$ \\
\hline $\mathrm{Al}$ & $2 c$ & 0.333 & 0.666 & 0.250 & $0.61(9)$ & $\mathrm{Ga}$ & $2 c$ & 0.333 & 0.666 & 0.250 & $0.61(9)$ \\
\hline \multicolumn{12}{|c|}{$\mathrm{Ni}_{2}$ In-type hexagonal structure with space group $P 6_{3} / m m c^{\mathrm{c}}$} \\
\hline $\mathrm{Mn}$ & $2 a$ & 0.000 & 0.000 & 0.000 & $2.39(8)$ & $\mathrm{Mn}$ & $2 a$ & 0.000 & 0.000 & 0.000 & 2.34(8) \\
\hline $\mathrm{Ni}$ & $2 d$ & 0.333 & 0.666 & 0.750 & $0.05(2)$ & $\mathrm{Ni}$ & $2 d$ & 0.333 & 0.666 & 0.750 & $0.10(2)$ \\
\hline $\mathrm{Ge}$ & $2 c$ & 0.333 & 0.666 & 0.250 & $0.92(9)$ & $\mathrm{Ge}$ & $2 c$ & 0.333 & 0.666 & 0.250 & $0.45(9)$ \\
\hline $\mathrm{Al}$ & $2 c$ & 0.333 & 0.666 & 0.250 & $0.92(9)$ & $\mathrm{Ga}$ & $2 c$ & 0.333 & 0.666 & 0.250 & $0.45(9)$ \\
\hline
\end{tabular}

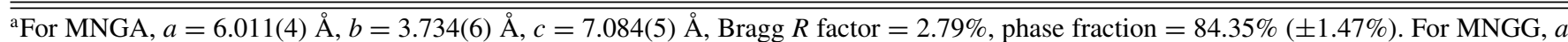
$=6.017(9) \AA, b=3.725(9) \AA, c=7.086(3) \AA$, Bragg $R$ factor $=4.59 \%$, phase fraction $=98.05 \%( \pm 0.48 \%)$.

${ }^{\mathrm{b}}$ For MNGA, $a=4.085(8) \AA, c=5.350(3) \AA, \quad \gamma=120^{\circ}$, Bragg $R$ factor $=5.27 \%$, phase fraction $=14.04 \%( \pm 0.41 \%)$. For MNGG, $a=$ $4.060(1) \AA, c=5.347(6) \AA, \gamma=120^{\circ}$, Bragg $R$ factor $=6.09 \%$, phase fraction $=1.37 \%( \pm 0.05 \%)$.

${ }^{\mathrm{c}}$ For MNGA, $a=4.093(1) \AA, c=5.415(8) \AA, \gamma=120^{\circ}$, Bragg $R$ factor $=5.07 \%$, phase fraction $=98.39 \%( \pm 0.95 \%)$. For MNGG, $a=$ 4.095(4) $\AA, c=5.420(7) \AA, \gamma=120^{\circ}, \operatorname{Bragg} R$ factor $=5.19 \%$, phase fraction $=99.40 \%( \pm 1.01 \%)$.

reflections, observed for the presently studied alloys, as a function of $T$ [see Figs. 6(b) and 6(d)]. These $(000)_{\mathrm{o}}^{ \pm}$and $(001)_{\mathrm{h}}$ magnetic reflections show one-to-one correspondence with the orthorhombic and hexagonal phase fractions, respectively. Higher values of the magnetic transition point compared to the MPT for both $\mathrm{Al}$ and Ge doped alloys provide us with a small window with a significantly large fraction of magnetically ordered hexagonal phases. Such behavior helped us to correctly determine the true nature of the magnetically ordered hexagonal phase. Our refinement of the NPD data also indicates a monotonic increase in the propagation vector with increasing sample $T$ for the presently studied

TABLE II. Fourier coefficients of the helicoidal magnetic structure of the $\mathrm{MnNiGe}_{0.9} \mathrm{Al}_{0.1}$ [propagation vector $\mathbf{k}=(0.2066(2), 0,0)$ ] and $\mathrm{MnNiGe}_{0.928} \mathrm{Ga}_{0.072}$ [propagation vector $\mathbf{k}=(0.2088(3), 0,0)$ ] alloys, refined at $T=1.5 \mathrm{~K}$. The magnetic structure was defined as $(R \mathbf{v}+$ $i I \mathbf{w}) e^{-2 \pi i(\mathbf{k} \cdot \mathbf{t}+\varphi)}$, where $R$ and $I$ are the real and imaginary parts of the Fourier coefficients, respectively, $\mathbf{t}$ is the lattice translation, and $\varphi$ is the magnetic phase. $\mathbf{v}$ and $\mathbf{w}$ are unit vectors defining the plane of the spin rotation (the $b c$ plane in the present case).

\begin{tabular}{|c|c|c|}
\hline \multicolumn{3}{|c|}{$\mathrm{MnNiGe}_{0.9} \mathrm{Al}_{0.1}, T=1.5 \mathrm{~K}$} \\
\hline $\mathrm{Mn}_{11}(0.0321,0.2500,0.1824)$ & $3.08(3)$ & $0.000(0)$ \\
\hline $\mathrm{Mn}_{21}(0.4678,0.7500,0.6824)$ & $3.08(3)$ & $0.113(3)$ \\
\hline $\mathrm{Mn}_{22}(0.9678,-0.2500,-0.1824)$ & $3.08(3)$ & $0.226(6)$ \\
\hline $\mathrm{Mn}_{12}(0.5326,0.2500,0.3175)$ & $3.28(9)$ & $0.104(4)$ \\
\hline $\mathrm{Mn}_{21}(0.4674,0.7500,0.6824)$ & $3.28(9)$ & $0.114(4)$ \\
\hline $\mathrm{Mn}_{22}(0.9674,-0.2500,-0.1824)$ & $3.28(9)$ & $0.228(8)$ \\
\hline
\end{tabular}



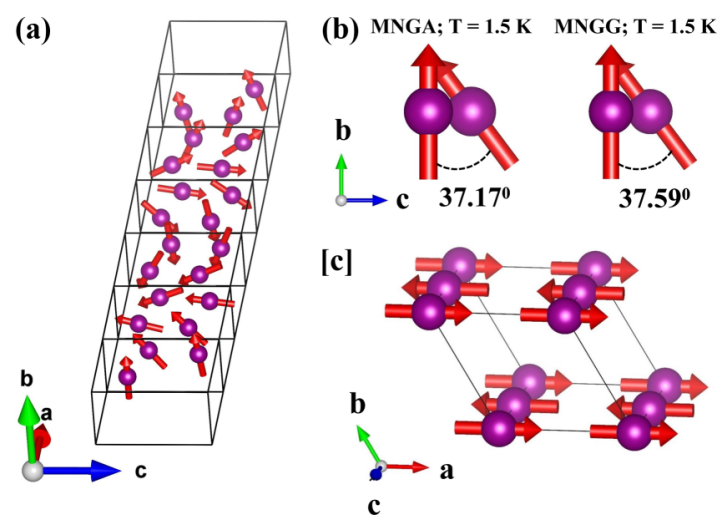

FIG. 3. (a) Incommensurate magnetic structure with helical modulation at $1.5 \mathrm{~K}$. (b) The angle between two adjacent magnetic spins observed for the MNGA and MNGG alloys. (c) The commensurate antiferromagnetic structure. For clarity, only the magnetic atoms, $\mathrm{Mn}$, are shown here.

alloys (see Fig. 7). This is due to the shift in the position of the magnetic reflections originating from the orthorhombic martensitic phase. During the refinement of the NPD data, we carefully checked for the presence of any FM ordering in these alloys, as previous reports claimed the presence of such ordering (based on the macroscopic measurements) in the hexagonal phase of this type of Ge-site doped MnNiGe alloys [11-14,32]. However, we have not observed any trace of ferromagnetic ordering in the presently studied alloys (at least in the absence of an external magnetic field).

\section{DISCUSSION AND CONCLUSION}

We performed a systematic investigation of the magnetic structure of the Ge-site doped MnNiGe alloys with the nominal compositions $\mathrm{MnNiGe}_{0.9} \mathrm{Al}_{0.1}$ and $\mathrm{MnNiGe}_{0.928} \mathrm{Ga}_{0.072}$
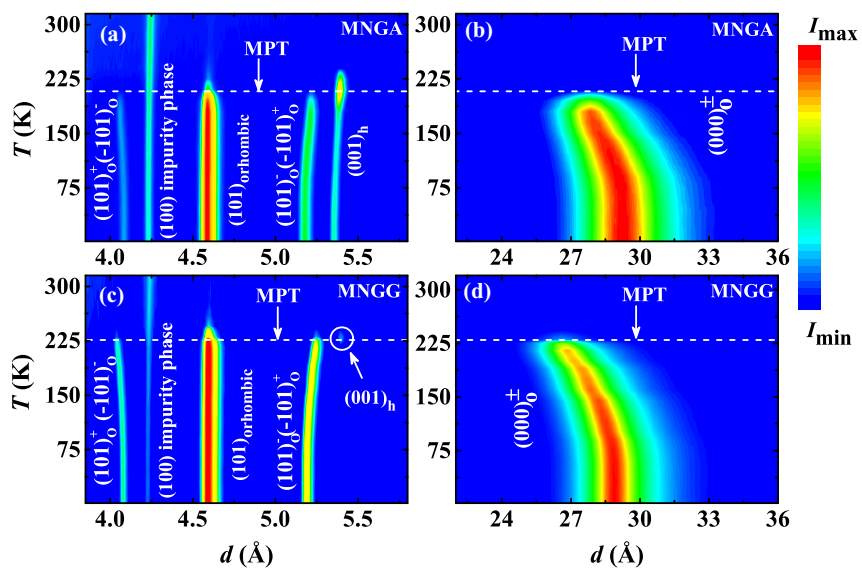

FIG. 4. Contour plots of the temperature evolution of the $(101)_{\mathrm{o}}^{+}$, $(-101)_{\mathrm{o}}^{-},(101)_{\mathrm{o}}^{-},(-101)_{\mathrm{o}}^{+},(000)_{\mathrm{o}}^{ \pm}$, and $(001)_{\mathrm{h}}$ magnetic satellite reflections (a) and (b) for MNGA and (c) and (d) for MNGG. Here, the suffixes "o" and "h" stand for orthorhombic and hexagonal phases, respectively. The right-hand scale bar indicates the normalized intensity. The martensitic phase transition (MPT) is indicated by the white dashed lines.

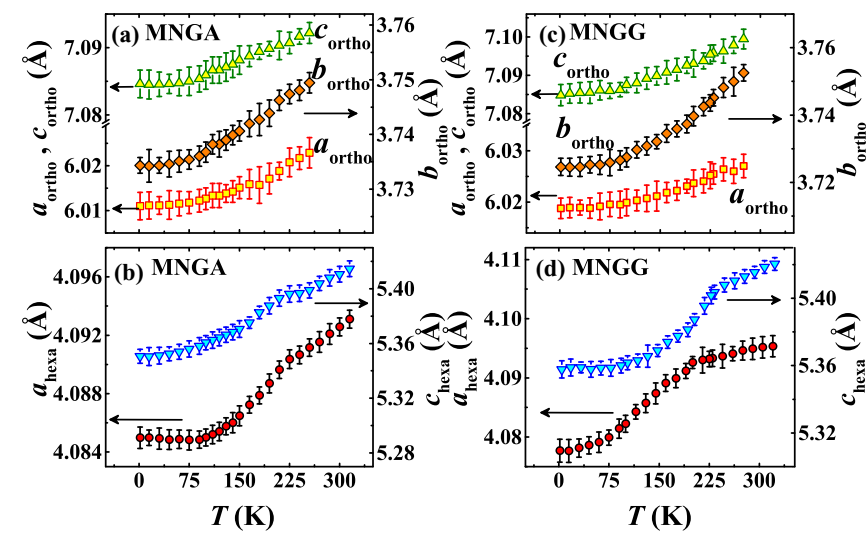

FIG. 5. (a) and (c) The temperature $T$ variation of orthorhombic lattice parameters for the MNGA and MNGG alloys, respectively. (b) and (d) $T$ variation of the hexagonal lattice parameters for the MNGA and MNGG alloys, respectively.

through detailed neutron powder diffraction measurements at different constant temperatures. Doping of both smaller size $\mathrm{Al}$ and larger size Ga in the Ge site of the MnNiGe alloy results in a decrease in this alloy's lattice parameters. This is consistent with the previously reported data $[11,13]$. In addition, for both these cases, the valance electron concentration ( $e / a$ ratio) decreases with doping. These effects play a crucial role in the significant decrease in the MPT compared to the undoped MnNiGe alloy. Apart from the reduction of MPT, the present work revealed (i) helically modulated incommensurate AFM ordering of the low- $T$ martensite phase, (ii) a gradual increase in the incommensurate propagation vector with increasing sample temperature, (iii) a commensurate AFM ordering of the high- $T$ austenite phase, and (iv) the absence of any ferromagnetic ordering in the absence of an external magnetic field. Similar incommensurate AFM ordering with helical modulation has also been reported for $\mathrm{Si}$, $\mathrm{Ti}$, and $\mathrm{Cr}$ doped $\mathrm{MnNiGe}$ alloys [25-28]. Along the direction
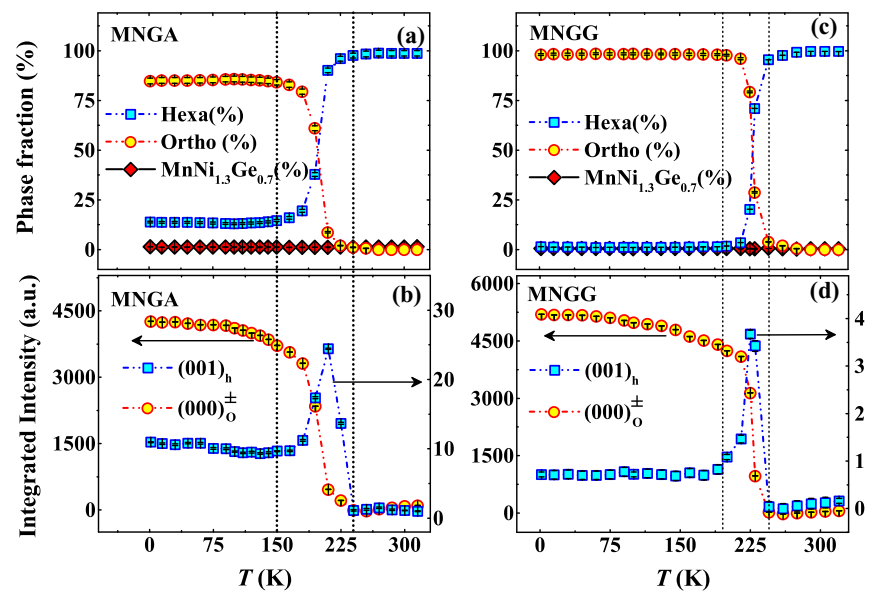

FIG. 6. (a) and (c) Temperature $T$ variation of the orthorhombic, hexagonal, and $\mathrm{MnNi}_{1.3} \mathrm{Ge}_{0.7}$ phases present in the MNGA and MNGG alloys, respectively. (b) and (d) Integrated intensities of $(000)_{\mathrm{o}}^{ \pm}$and $(001)_{\mathrm{h}}$ magnetic satellite reflections as a function of $T$ for the MNGA and MNGG alloys, respectively. 


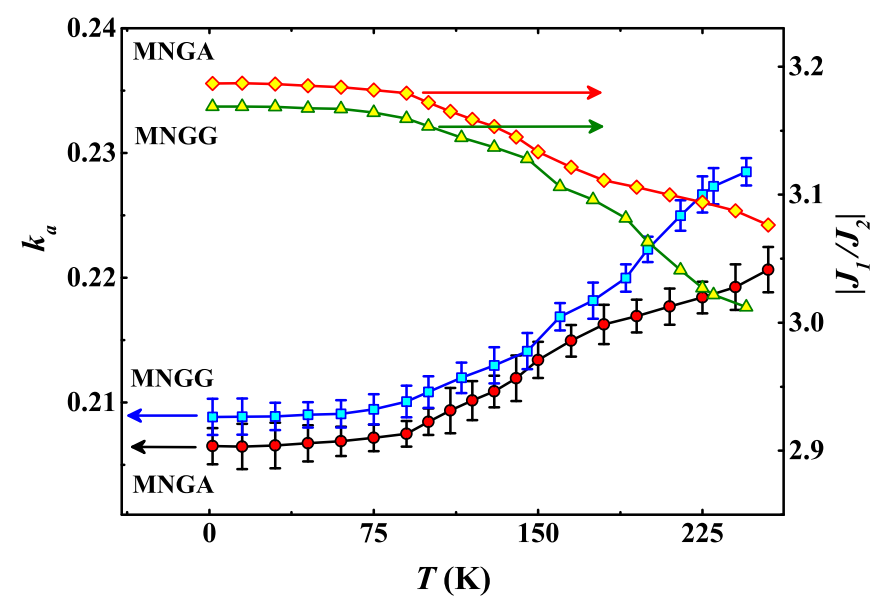

FIG. 7. Temperature variation of the incommensurate propagation vector $k_{a}$ and the ratio of nearest-neighbor to next-nearestneighbor exchange interaction for both the MNGA and MNGG alloys.

of the propagation vector, we observed rotation of the moment direction by a certain angle $(\alpha)$ while moving from one Mn layer to another. Competing exchange interactions among the $\mathrm{Mn}$ atoms of different layers are mainly responsible for such moment rotation along the propagation vector and hence for the helical magnetic arrangement. Also, we have noticed that the angle of moment rotation $\alpha$ depends strongly on the sample temperature. The value of $\alpha$ was found to be $37.17^{\circ}$ and $37.59^{\circ}$ for the MNGA and MNGG alloys, respectively, at $1.5 \mathrm{~K}$. With increasing sample $T$, the value of $\alpha$ increases and becomes $39.72^{\circ}$ (at $255 \mathrm{~K}$ ) and $41.13^{\circ}$ (at $245 \mathrm{~K}$ ) for the $\mathrm{Al}$ and Ga doped alloys, respectively. Previous work on the NPD study of the undoped $\mathrm{MnNiGe}$ alloy reported a rotation of the axis of the helical magnetic structure from the $b c$ plane to the $a$ axis with increasing sample temperature [24]. However, such a change in the axis of the helix is not observed for the presently studied alloys. Apart from the magnetic equiatomic alloys, a similar kind of helically modulated magnetic structure has also been reported for the rare earth elements Tb, Dy, and Ho, where competing interactions are mainly responsible for such a modulated structure [33].

The axial next-nearest-neighbor Ising model was sometimes used to explain such a helically modulated magnetic arrangement [27,28,34,35]. This model incorporates anisotropic competing exchange interaction with nearest-neighbor ferromagnetic interaction in a plane perpendicular to the helical magnetic structure's modulation axis [27,28,34,35]. On the other hand, nearest-neighbor FM and next-nearest-neighbor AFM interactions along the modulation axis lead to the helical arrangement of magnetic spins perpendicular to the FM plane. According to Enz [35], the stability criteria for achieving a stable helical structure can be written as $\cos \alpha=-\frac{J_{1}}{4 J_{2}}$, where $J_{1}$ and $J_{2}$ signify the FM (Mn-Mn distance $\left.=a / 2\right)$ and AFM (Mn-Mn distance $=a$ ) interactions, respectively, along the modulation axis. From the refinement analysis of the NPD data, recorded at different constant $T$, we have estimated the values of $\alpha$ for both alloys. Such values of $\alpha$ show good agreement with the relation $\alpha=180^{\circ} \times k_{a}$ used by Penc et al. as well $[27,28]$. Using the stability criteria of the helical mod- ulation and values of $\alpha$ obtained from NPD analysis, we have estimated and plotted the values of the $\frac{J_{1}}{J_{2}}$ ratio as a function of $T$ for both alloys (see Fig. 7). We observed a sluggish decrease in the $\frac{J_{1}}{J_{2}}$ ratio up to $75 \mathrm{~K}$, followed by a faster decrease with a further increase in $T$. Such behavior of the $\frac{J_{1}}{J_{2}}$ ratio confirms the increasing strength of the next-nearestneighbor AFM interaction compared to the nearest-neighbor FM interaction. The observed behavior is again similar to the $\mathrm{Mn} / \mathrm{Ni}$-site doped MnNiGe alloys [25-28].

Observation of a commensurate-type antiferromagnetically ordered hexagonal phase [with $\mathbf{k}_{\mathbf{h}}=(0,0,0)$ ] is one of the critical findings of the present work. Previous and present results indicate a considerable decrease in MPT temperature in Ge-site doped MnNiGe alloys. In addition, Mn chains and the $\mathrm{Ni}-6 \mathrm{Mn}$ environment remain unchanged with Ge-site doping. Such a large reduction in MPT and an intact Mn environment allowed the hexagonal phase to order antiferromagnetically. However, based on the bulk magnetic data, ordering of the hexagonal phase was previously identified as FM in nature. Incomplete MPT for both of the presently studied alloys (especially for MNGA with the presence of $14.04 \%$ hexagonal phase at $1.5 \mathrm{~K}$ ) provided the opportunity to investigate the true magnetic character of the magnetically ordered hexagonal phase down to the lowest temperature of the measurement ( $1.5 \mathrm{~K}$ for the present case). The presence of magnetic satellite reflections and the absence of any intensity mismatch between the observed and calculated intensities of the nuclear reflections of the hexagonal phase below the ordering temperature confirm the AFM ordering of the hexagonal phase and the absence of any FM order in the presently studied alloys (at least in the absence of an external magnetic field).

In conclusion, the present work, based on the NPD analysis of two Ge-site doped MnNiGe alloys with the nominal compositions $\mathrm{MnNiGe}_{0.9} \mathrm{Al}_{0.1}$ and $\mathrm{MnNiGe}_{0.928} \mathrm{Ga}_{0.072}$, reveals the true magnetic structure of both the hexagonal and orthorhombic phases present in these alloys. A helically modulated incommensurate AFM ordering, with its axis lying along the $a$ axis, of the low- $T$ orthorhombic phase is clear from the NPD data recorded in the absence of an external magnetic field. With increasing sample temperature, an apparent increase in the incommensurate propagation vector and hence in the AFM strength have been observed for both alloys. On the other hand, no change in the helix axis has been observed throughout the magnetically ordered region. Interestingly, the high- $T$ hexagonal phase is found to order antiferromagnetically with a commensurate structure for both alloys (below a specific temperature, depending on the composition).

Neutron diffraction data were recorded on the WISH diffractometer at the ISIS Neutron and Muon Source. Information on the data can be accessed through STFC ISIS Facility [36].

\section{ACKNOWLEDGMENTS}

S.C. would like to thank the Science and Engineering Research Board (SERB), DST-India, for financial support (Project No. CRG/2020/000670). S.C.D. and S.C. would also like to thank DST-India (Grant No. SR/NM/Z-07/2015) for access to the experimental facility and financial support to 
carry out the experiments, and the Jawaharlal Nehru Centre for Advanced Scientific Research (JNCASR) for managing the DST-India project. S.C.D. would like to thank DST-India for his Inspire Fellowship (Grant No. IF160587). J.S. would like to thank the European Union's Horizon 2020 research and innovation program under Marie Skłodowska-Curie Grant Agreement No. 665593 awarded to the Science and Technology Facilities Council.
[1] L. Morellon, J. Stankiewicz, B. García-Landa, P. A. Algarabel, and M. R. Ibarra, Appl. Phys. Lett. 73, 3462 (1998).

[2] P. A. Algarabel, M. R. Ibarra, C. Marquina, A. del Moral, J. Galibert, M. Iqbal, and S. Askenazy, Appl. Phys. Lett. 66, 3061 (1995).

[3] V. Sechovský, L. Havela, K. Prokeš, H. Nakotte, F. R. de Boer, and E. Brück, J. Appl. Phys. 76, 6913 (1994).

[4] K. A. Gschneidner, Jr., V. K. Pecharsky, and A. O. Tsokol, Rep. Prog. Phys. 68, 1479 (2005).

[5] R. Kainuma, Y. Imano, W. Ito, Y. Sutou, H. Morito, S. Okamoto, O. Kitakami, K. Oikawa, A. Fujita, T. Kanomata, and K. Ishida, Nature (London) 439, 957 (2006).

[6] T. Krenke, E. Duman, M. Acet, E. F. Wassermann, X. Moya, L. Mañosa, A. Planes, E. Suard, and B. Ouladdiaf, Phys. Rev. B 75, 104414 (2007).

[7] L. Morellon, P. A. Algarabel, M. R. Ibarra, J. Blasco, B. GarcíaLanda, Z. Arnold, and F. Albertini, Phys. Rev. B 58, R14721 (1998).

[8] C. L. Zhang, D. H. Wang, Q. Q. Cao, Z. D. Han, H. C. Xuan, and Y. W. Du, Appl. Phys. Lett. 93, 122505 (2008).

[9] C. Zhang, D. Wang, Q. Cao, S. Ma, H. Xuan, and Y. Du, J. Phys. D 43, 205003 (2010).

[10] P. Dutta, S. Pramanick, D. Venkateshwarlu, V. Ganesan, S. Majumdar, D. Das, and S. Chatterjee, Europhys. Lett. 108, 17012 (2014).

[11] T. Samanta, I. Dubenko, A. Quetz, S. Temple, S. Stadler, and N. Ali, Appl. Phys. Lett. 100, 052404 (2012).

[12] A. Quetz, T. Samanta, I. Dubenko, M. J. Kangas, J. Y. Chan, S. Stadler, and N. Ali, J. Appl. Phys. 114, 153909 (2013).

[13] C. L. Zhang, Z. D. Han, B. Qian, H. F. Shi, C. Zhu, J. Chen, and T. Z. Wang, J. Appl. Phys. 114, 153907 (2013).

[14] E. Liu, Y. Du, J. Chen, W. Wang, H. Zhang, and G. Wu, IEEE Trans. Magn. 47, 4041 (2011).

[15] P. Dutta, S. Pramanick, S. Majumdar, D. Das, and S. Chatterjee, J. Magn. Magn. Mater. 395, 312 (2015).

[16] E. Liu, W. Wang, L. Feng, W. Zhu, G. Li, J. Chen, H. Zhang, G. Wu, C. Jiang, H. Xu, and F. de Boer, Nat. Commun. 3, 873 (2012).
[17] P. Dutta, S. Pramanick, V. Singh, D. T. Major, D. Das, and S. Chatterjee, Phys. Rev. B 93, 134408 (2016).

[18] P. Dutta, S. Pramanick, D. Das, and S. Chatterjee, J. Phys. D 49, 385001 (2016).

[19] K. Mandal, P. Dutta, P. Dasgupta, S. Pramanick, and S. Chatterjee, J. Phys. D 51, 225004 (2018).

[20] K. Mandal, S. C. Das, P. Dutta, S. Pramanick, P. Dasgupta, and S. Chatterjee, J. Appl. Phys. 124, 215904 (2018).

[21] K. Mandal, S. C. Das, P. Dutta, S. Pramanick, and S. Chatterjee, J. Alloys Compd. 822, 153454 (2020).

[22] S. C. Das, K. Mandal, P. Dutta, S. Pramanick, and S. Chatterjee, J. Phys. D 51, 065002 (2018).

[23] S. C. Das, K. Mandal, P. Dutta, S. Pramanick, and S. Chatterjee, J. Magn. Magn. Mater. 465, 44 (2018).

[24] W. Bażela, A. Szytuła, J. Todorović, Z. Tomkowicz, and A. Zięba, Phys. Status Solidi A 38, 721 (1976).

[25] W. Bażela, A. Szytuła, J. Todorović, and A. Zięba, Phys. Status Solidi A 64, 367 (1981).

[26] W. Bażela and A. Szytuła, Phys. Status Solidi A 66, 45 (1981).

[27] B. Penc, A. Hoser, S. Baran, R. Duraj, M. Marzec, T. J. Gołaąb, A. Szytuła, V. Dyakonov, N. Nedelko, A. Sivachenko, K. Dyakonov, W. Bażela, and H. Szymczak, Phase Transitions 91, 1107 (2018).

[28] B. Penc, A. Hoser, S. Baran, and A. Szytuła, Phase Transitions 91, 118 (2018).

[29] L. C. Chapon, P. Manuel, P. G. Radaelli, C. Benson, L. Perrott, S. Ansell, N. J. Rhodes, D. Raspino, D. Duxbury, E. Spill, and J. Norris, Neutron News 22, 22 (2011).

[30] V. F. Sear, Neutron News 3, 26 (1992).

[31] J. Rodríguez-Carvajal, Phys. B (Amsterdam, Neth.) 192, 55 (1993).

[32] Y. V. Kuźma, Y. M. Teslyuk, and E. I. Gladyshevskii, J. Struct. Chem. 3, 143 (1962).

[33] T. Chattopadhyay, Science 264, 226 (1994).

[34] A. Herpin, P. Meriel, and J. Villain, J. Phys. Radium 21, 67 (1960).

[35] U. Enz, J. Appl. Phys. 32, 22S (1961).

[36] S. Chatterjee et al., (2019), doi:10.5286/ISIS.E.RB1968024. 